\title{
Microbial Patterns in Rumen Are Associated with Gain of Weight in Beef Cattle
}

\author{
Anderson Santos de Freitas ${ }^{1,+}$, Diego Bitencourt de David ${ }^{2,+}$, Beatriz Midori Takagaki ${ }^{1}$ and Luiz \\ Fernando Würdig Roesch 1,* \\ 1 Centro Interdisciplinar de Pesquisas em Biotecnologia - CIP-Biotec, Campus São Gabriel, Universidade \\ Federal do Pampa, São Gabriel, Rio Grande do Sul, Brasil. \\ 2 Departamento de Diagnóstico e Pesquisa Agropecuária - DDPA, Secretaria Estadual da Agricultura, \\ Pecuária e Desenvolvimento Rural - SEADPR/RS, São Gabriel, Rio Grande do Sul, Brasil. \\ + These authors have contributed equally to this work. \\ * Correspondence: luizroesch@unipampa.edu.br
}

\begin{abstract}
Ruminal microorganisms play a pivotal role in cattle nutrition. The discovery of the main microbes responsible for enhancing the gain of weight in beef cattle might be used in therapeutic approaches to increase animal performance and cause less environmental damages. Here, we examined differences in bacterial and fungal composition of rumen samples of Braford heifers raised in a natural grassland from Pampa Biome in Brazil. We aimed to detect microbial patterns in the rumen that could be correlated with the gain of weight. 16S and ITS1 genes were amplified from ruminal samples and sequenced to identify the closest microbial relatives within the microbial communities. A predictive model based on microbes responsible for the gain of weight was build and further tested using the entire dataset. The model detected a set of microorganisms associated with animals in the high gain of weight group, including the bacterial taxa RFN20, Prevotella, Anaeroplasma and RF16 and the fungal taxa Aureobasidium, Cryptococcus, Sarocladium, Pleosporales and Tremellales. Most of these organisms have been correlated to the production of substances that improve the ruminal digestion process. These findings provide new insights about cattle nutrition and suggest the use of these microbes to improve beef cattle breeding.
\end{abstract}

Keywords: bacteria; fungi; livestock; microbiome; next generation sequencing.

\section{Introduction}

Microorganisms from all domains of life are associated with cattle nutrition. Bacteria, Archaea and micro Eukaryotes are present across the entire gastrointestinal tract (GIT) of these animals. They digest complex molecules, such as cellulose and lignin, and help the absorption of water and nutrients [1,2]. Microbes from the rumen, in particular, are crucial for this process, once the rumen acts as a fermentation chamber hosting microbial fermentation of the food ingested by the cattle. The rumen environment is responsible for about 50 to 65 percent of starch and soluble sugars utilized by an animal and produces the main food and energy sources for bovines (i.e. proteins, volatile fatty acids, and synthesis of $\mathrm{B}$ and $\mathrm{K}$ vitamins) [3,4].

Since mimicking the rumen conditions in laboratories to isolate those microbes is a challenging task, the rising of culture-free techniques, mainly Next Generation Sequencing (NGS), has facilitated the study of these microbes. Over the achievements accumulated by classic culture-based approaches, NGS has provided a wide range of knowledge about rumen microbes and its behavior in the last decades [5,6]. Several studies, using different techniques and bovine races, have been uncovered the structure of the rumen environment and pointing to a consensus ruminal core microbiome across a large geographical range $[7,8]$. This core is dominated by the bacterial phylum Bacteroidetes, mainly composed by Prevotella species, either in Brazilian Nelore bovines [9] or in Holstein bull calves from Canada [1], for example. Indeed, this predominance of Bacteroidetes and its Prevotella species is extensively described by reports that denote these taxa as important 
microorganisms for digestion of organic matter in the rumen, being considered as biomarkers for good ruminal microbiota [10]. With respect to fungi, the ruminal microbial core includes a range of anaerobic genera, mainly from Neocallimastigomycota, Basidiomycota and Ascomycota phyla [11,12].

Although geographical standards of ruminal microbiota are known, previous works also support that external aspects like diet may influence the rumen microbial composition. Animals submitted to a low-forage diet, for example, showed a higher abundance of Entodinium and Prevotella species [13] whereas animals with induced sub-acute ruminal acidosis (SARA) presented an increase of rare fungal taxa [14]. Similarly, different microbial composition affect production chains, such as cows with high milk yield, which presented higher levels of Prevotella [15] and the correlation of milk-fat yield to a higher Firmicutes/Bacteroidetes ratio in rumen [16].

All this knowledge comes to meet the growing need for food supply and the concern about climate changes in the world. These areas may be benefitted by new insights about how to optimize cattle breeding with more production and less environmental damages [17]. Alongside, the ruminal microbiology has historically failed in optimize animal performance by microbial information and therapies. Much of this is due to the lack of knowledge about how microorganisms act biochemically through the digestion processes, a situation that is being reveled by novel high throughput techniques [5].

In this context, and considering the economic importance of cattle breeding as well as the constant search for improvements in production, we purpose ourselves to find microbial patterns in rumen that could be correlated with gain of weight, the key topic in beef cattle breeding. Here, we tested the differences of microbial communities in a set of heifers raised in natural grasslands from the Pampa Biome. Heifers with a high daily gain of weight were compared with the ones with a low daily gain. Our hypothesis was that animals with a higher gain of weight might have microbial patterns that can explain a better digestion process and, consequently, an enhanced gain of weight.

\section{Materials and Methods}

\subsection{Cattle management and experimental design}

In this work, we attempted to build a predictive model for microbial biomarkers of enhanced gain of weight using a subset of rumen samples and further test the model using the entire dataset. For this, we selected animals in the same natural grasslands from the Pampa Biome and calculated the average daily weight gain (ADG) for all of them. Based on ADGs, we selected the upper and lower quartile groups of weight gain and compared them for composition of both bacteria and fungi, targeting microorganisms that could be increased in one of the groups.

The animals were raised by the Departamento de Diagnóstico e Pesquisa Agropecuária (DDPA, SEAPI-RS), located in São Gabriel, RS, Brazil. All animal management and research procedures were approved by the Animal Welfare Committee of the State Foundation of Agricultural Research (FEPAGRO) registered under the number 15/14. We sampled seventeen Braford heifers (36 months, $343 \pm 30 \mathrm{~kg}$ ) randomly distributed in paddocks with natural grasslands from the Pampa Biome for more than two years. These animals were submitted to a continuous grazing stock method, with a forage allowance of $12 \mathrm{~kg}$ of dry matter $/ 100 \mathrm{~kg}$ of live weight adjusted by a put and take animals method. The pasture structure during the sampling period is described in Table 1. The native pastures include mainly the species Eryngium horridum, Vernonia nudiflora, Erianthus angustifolius, Eupatorium bunifolium, Paspalum notatum, Eragrostis plana, Axonopus affinis, Paspalum umbrosum, Desmodium incanum and Paspalum plicatulum. 
Table 1. Description of the pasture structure during the sampling period.

\begin{tabular}{ccc}
\hline Measurements & Mean & Standard Deviation \\
\hline Herbage accumulation (kg/day) & 17.28 & 1.18 \\
Pasture height $(\mathrm{cm})$ & 9.74 & 1.44 \\
Herbage mass $(\mathrm{kg})$ & 2530 & 240 \\
$\begin{array}{c}\text { Forage allowance }([\mathrm{kg} \text { of } \\
\text { DM]/[100 kg of LW]) }\end{array}$ & 11.43 & 2.98 \\
*Eragorstis plana cover $(\%)$ & 14 & 6.68 \\
\hline
\end{tabular}

*Exotic gramineous plant considered an aggressive invasive plant with low nutritional quality and high resistance to mechanic traction.

We monitored the weight gain for 60 days, with the sampling occurring in the middle of this period (30 days) in the autumn, aiming to minimize effects of extreme climate conditions. Based on this monitoring, we estimate the average daily weight gain (ADG) for each animal and proceed to downstream analysis.

\subsection{Rumen sampling strategy and diet quality measurement}

We collected ruminal fluid from 17 heifers shortly after the morning grazing cycle (approximately between 9:00 and 12:00 h). Samples of the ruminal fluid (100 ml) were collected through an esophageal probe equipped with a vacuum system. For each animal, a sterilized set of hoses and kitassato flasks were used in order to avoid cross-contamination. Ruminal liquid samples were immediately stored in $50 \mathrm{~mL}$ tubes properly identified and stored in ice-cold box for transportation to the laboratory. Approximately two hours after the collection, the tubes were frozen at $-80^{\circ} \mathrm{C}$. Before sampling the ruminal fluid, a fecal sample was collected via rectum using sterile gloves. This fraction was designated for analysis of diet quality as described below.

Analysis of relationship between fecal protein concentration and diet digestibility provided dietary quality estimation by methods previously described by Lancaster [18] and Rosa and colleagues [19]. Briefly, fecal samples were oven dried with forced air circulation at $55^{\circ} \mathrm{C}$ for 72 hours. After partially dried and grounded, feces were subjected to dry matter determination after drying at $105^{\circ} \mathrm{C}$ for 12 hours (Easley et al., 1965). The organic matter was determined by flaring at $550^{\circ} \mathrm{C}$ and crude protein (CP) was determined by the Kjeldahl method (Kirk, 1950). The concentration of fecal CP in the organic matter (CPf:g/kg.OM) was used in the equations (1), (2) and (3) described below to estimate organic matter digestibility (OMD), protein concentration in the diet (PC) and daily protein consumption (CP Intake). The equations were constructed specifically for native grasslands of Pampa Biome by using the Rosa's et al. methods (Rosa et al., n.d.):

$$
\begin{gathered}
\text { OMD }=0.942-38,619 / \text { fecalCP } \\
\text { PC }=1.346^{*}(\text { fecalCP })-47.63(2) \\
\text { CPintake }=8.0728^{*}(\text { fecalCP })-347.38
\end{gathered}
$$

\subsection{DNA extraction and PCR amplification}

For DNA extraction, we used $1 \mathrm{ml}$ of homogenized ruminal fluid samples from each animal. DNA extraction was performed with the PowerSoil@DNA kit (MoBio, USA) according to the manufacturer's instructions. Concentrations and purity of the DNA were determined using the NanoVue ${ }^{\mathrm{TM}}$ spectrophotometer (GE Healthcare, USA). All DNA samples were stored at $-20^{\circ} \mathrm{C}$ until use for the PCR reactions. 
The amplification, sequencing and processing of the data were performed according to the recommendations of the Brazilian Microbiome Project - available at http://www.brmicrobiome.org [20]. For each sample, the $v 4$ region from the $16 \mathrm{~S}$ gene was amplified by using the $515 \mathrm{~F} / 806 \mathrm{R}$ primers [21] for amplification of microorganisms of the Bacteria and Archaea domain simultaneously. The primer was synthesized together with the A-Key (5'CCATCTCATCCCTGCGTGTCTCCGACTCAG'3) P1-Key (5'CCTCTCTATGGGCAGTCGGTGAT'3) adapters to obtain a sequence composed for A-barcode-806R and P1-515F adapter and primers. A similar approach was used to the internal transcribed spacer region (ITS1) which was amplified by means of the ITS1F/ITS2R primers [22-24] for the amplification of microorganisms from the Fungi kingdom in each sample. Twelve known bases (barcodes) were added to the 5 ' region of the primers, which were used to identify the origin of each sequence, according to the methodology proposed by Hamady and collaborators [25] either for bacteria and fungi.

PCR reactions were performed for each DNA isolated from the samples with a different barcode per reaction. For bacteria, each of the $25 \mathrm{~mL}$ of PCR mixture consisted of $2 \mathrm{U}$ of Platinum Taq DNA High Fidelity Polymerase (Invitrogen, Carlsbad, CA, United States), 4 uL 10X High Fidelity PCR Buffer, $2 \mathrm{mM}$ MgSO4, $0.2 \mathrm{mM}$ dNTP's, $0.1 \mathrm{mM}$ of both the $806 \mathrm{R}$ barcoded primer and the $515 \mathrm{~F}$ primer, $25 \mathrm{ug}$ of Ultrapure BSA (Invitrogen, Carlsbad, CA, United States) and approximately $50 \mathrm{ng}$ of DNA template. The conditions used in these reactions were: $94^{\circ} \mathrm{C}$ for 2 minutes, 30 cycles at $94^{\circ} \mathrm{C}$ for $45 \mathrm{~s}$ for denaturation, $55^{\circ} \mathrm{C}$ for $45 \mathrm{~s}$ for annealing, and $72^{\circ} \mathrm{C}$ for 1 minute for extension; followed by $72{ }^{\circ} \mathrm{C}$ for 6 minutes of final extension. For fungi, each of the $50 \mu \mathrm{l}$ of PCR mixture consisted of $2 \mathrm{U}$ of Platinum Taq DNA High Fidelity Polymerase (Invitrogen, Carlsbad, CA, United States), 5 uL 10X High Fidelity PCR Buffer, $2 \mathrm{mM}$ MgSO4, $0.2 \mathrm{mM}$ dNTP's, $0.1 \mathrm{mM}$ of both the ITS2R barcoded primer and the ITS1F primer, 25 ug of Ultrapure BSA (Invitrogen, Carlsbad, CA, United States) and approximately $100 \mathrm{ng}$ of DNA template. The conditions used in the reactions for fungi were: $94^{\circ} \mathrm{C}$ for 2 minutes, 35 cycles at $94^{\circ} \mathrm{C}$ for $45 \mathrm{~s}$ for denaturation, $55^{\circ} \mathrm{C}$ for $60 \mathrm{~s}$ for annealing the primer oligonucleotides, and $72^{\circ} \mathrm{C}$ for 1.5 minute for extension; followed by $72^{\circ} \mathrm{C}$ for 10 minutes of final extension.

The resulting PCR products were purified with the Agencourt ${ }^{\circledR}$ AMPure ${ }^{\circledR}$ XP Reagent kit (Beckman Coulter, USA). The final concentration of the PCR product DNA was quantified using the Qubit Fluorometer kit (Invitrogen) following the manufacturer's recommendations. Finally, the PCR products combined into equimolar concentrations were used to library preparation win Ion One-Touch 2 System using Ion PGM Template OT2 400 Kit (Thermo Fisher Scientific, Waltham, MA, USA). Sequencing was performed in Ion PGM Sequencing 400 on the Ion PGM System using 318 Chip v2. The Raw sequences were deposited in the Sequence Read Archive (SRA), Biosample accession PRJNA599105.

\subsection{Statistical analysis}

After building the Operational Taxonomic Unity Table following the Brazilian Microbiome pipeline [26], the OTU tables were imported into the R enviroment and transformed by centered log-ratio to reflect the compositional nature of these type of data [27]. All analysis were performed independently for bacteria and fungi, but keeping the same parameters. Possible confounding variables were tested by a permutational analysis of variance (PERMANOVA) based on Euclidean distance with the vegan package [28]. Initial insights were provided by analysis of microbial richness. After, we tested the main differences between the communities from higher ADG and lower ADG groups by differential abundance of both phyla and OTUs using the ALDEx2 package [29]. We considered as differentially abundant all phyla or OTUs with effect size higher than 1 and a p-value calculated by the Welch's test lower than 0.1 [30]. Finally, we tested all OTUs matched as differentially abundant between groups as models of regression including all 17 samples aiming to validate our findings in the whole dataset. 


\section{Results}

\subsection{Subjects Characteristics}

Ten subjects were selected from an initial set of 17, by using the descriptive statistics summarized in Figure 1. These samples comprised the extreme quartiles of the dataset. We included samples with marginal values (matching with extreme quartile's borderlines, Figure 1) once this information kept the groups with widely different gains of weight and increased the power for downstream analyses (Table 2). Our goal was to build a model with maximum contrast between ADG groups keeping similar diet parameters among the tested animals. Both animal groups presented very similar rates of organic matter digestion, crude protein concentration in feces, estimated crude protein intake and live weight at the beginning of the experiment. On the other hand, the average dairy gain of weight was significantly different between groups, overcoming, in mean, differences of 400 grams of weight gain per day.

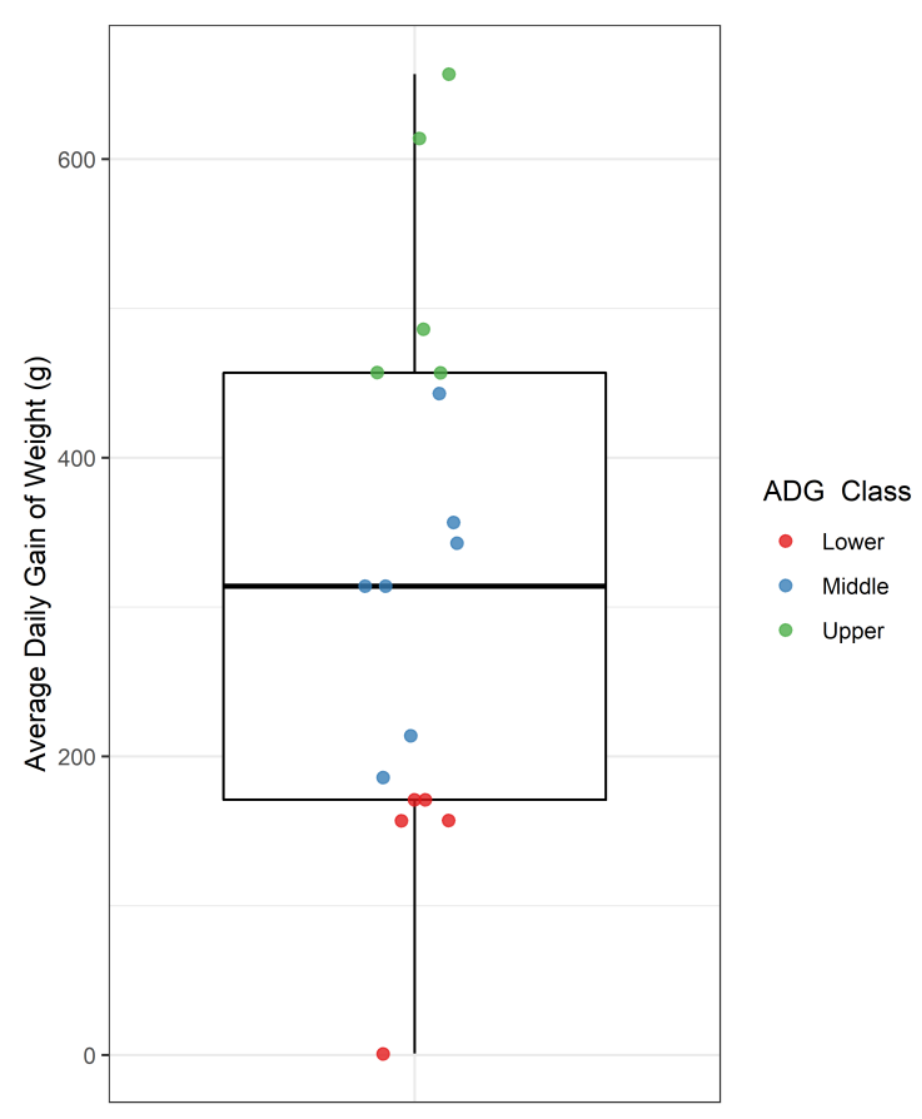

Figure 1. Distribution of average daily gain of weight (ADG) in the bovine samples. The first quartile was assigned to Lower ADG (red) and the fourth quartile to Higher ADG (green). Samples comprised in the second and third quartiles (blue) were classified as Middle ADG and were not included in differential abundance analysis. 
Table 2. Description of the diet variables from Higher Average Dairy Gain of Weight group (Higher ADG) versus the Lower Average Dairy Gain of Weight group (Lower ADG).

\begin{tabular}{lccc}
\hline \multicolumn{1}{c}{ Parameters } & Higher ADG & Lower ADG & p value \\
\hline Average Dairy Gain (g/day) & $534.2 \pm 42.2$ & $131.4 \pm 32.8$ & 0.01 \\
Organic Matter Digestion (\%) & $64.2 \pm 1.39$ & $65.3 \pm 0.67$ & 0.88 \\
Crude Protein Concentration & $117.4 \pm 8.52$ & $120.4 \pm 5.91$ & 1 \\
(g/kg OM) & & 1 \\
Crude Protein Intake (g/day) & $642.4 \pm 51.1$ & $660.3 \pm 35.5$ & 1 \\
Initial Live Weight (kg)* & $337.5 \pm 15.0$ & $337.3 \pm 15.6$ & 1 \\
\hline
\end{tabular}

Variables were summarized as average \pm SEM and compared using the Mann-Whitney-Wilcoxon test. Significant $\mathrm{p}$-values were highlighted in italic. $\mathrm{g}$ = grams; $\mathrm{kg}=$ kilograms; $\mathrm{OM}=$ organic matter. * Mean of live weight measured in the beginning of the experiment.

\subsection{Sequencing Report and Control for Confounding Variables}

A total of 289,137 high quality sequences were obtained from all 17 samples, with a mean of 12,072 bacterial sequences and 6,993 fungal sequences per sample. Good's coverage confirmed the number of sequences obtained were sufficient to describe the microbial community adequately. In average the coverage of the bacterial samples was higher than $95 \%$ and the coverage of the fungal samples was higher than $99 \%$.

To avoid possible false positives/negatives in downstream analysis, the presence of confounding variables [31] were tested by stratification. For doing this, a PERMANOVA analysis using all measured variables was performed and the results are shown in Table 3. No significant bias related to any possible confounding variable tested such as Eragorstis plana cover, percentage of crude protein in feces and the paddock effect was detected. In addition, analysis indicated that the bacterial communities in different ADG classes were strongly different $\left(R^{2}=0.14 ; p=0.03\right)$. On the other hand, no differences within the fungal community was observed between ADG classes.

Table 3. Detection of possible confounding variables associated with the lower and higher classes of Average Dairy Gain of Weight (ADG).

\begin{tabular}{lccc}
\hline & Confounding variables & $\mathbf{R}^{2}$ & p-value \\
\hline Bacteria & ADG Class & 0.14 & 0.03 \\
& Eragrostis plana Cover & 0.10 & 0.47 \\
& CP in feces & 0.20 & 0.37 \\
& Potrero & 0.35 & 0.09 \\
\hline Fungi & ADG Class & 0.22 & 0.30 \\
& Eragrostis plana Cover & 0.22 & 0.30 \\
& CP in feces & 0.42 & 0.47 \\
& Potrero & 0.57 & 0.60 \\
\hline
\end{tabular}

$\mathrm{R}^{2}$ and p-values were obtained by Permutational analysis of variance (PERMANOVA) based on the Euclidean dissimilarities applied to the Centered log-ratio (clr) transformed abundance of 16S rRNA sequences for bacterial/archaeal and fungal communities. Significant value is set in italics. $p$ values are based on 999 permutations. $\mathrm{ADG}=$ Average Daily Gain; $\mathrm{CP}=$ Crude Protein . 


\subsection{Overall description of bacterial and fungal communities}

We found 23 microbial phyla within all samples: 19 from Bacteria (Figure 2) and 4 from Fungi (Figure 3). Bacterial communities were dominated by species from the Bacteroidetes and Firmicutes, the only ones which presented values of centered log-ratio transformed abundance higher than 4 . Differential abundance analyses showed that phyla distribution between the ADG groups was very similar either in bacteria or in fungi (effect size $<1$ and p-value $>0.1$ for all tested phyla). Although we have found no significant difference among the bacterial phyla, some tendencies were observed: Tenericutes tended to be increased in the Higher ADG group (effect size $=0.55$, p-value $=0.29$ ) whereas WPS-2 tended to be increased in Lower ADG group (effect size $=0.70$, $\mathrm{p}$-value $=0.17$ ). In fungal communities, we were not able to detect any tendency depicting differences between groups (Figure 3).
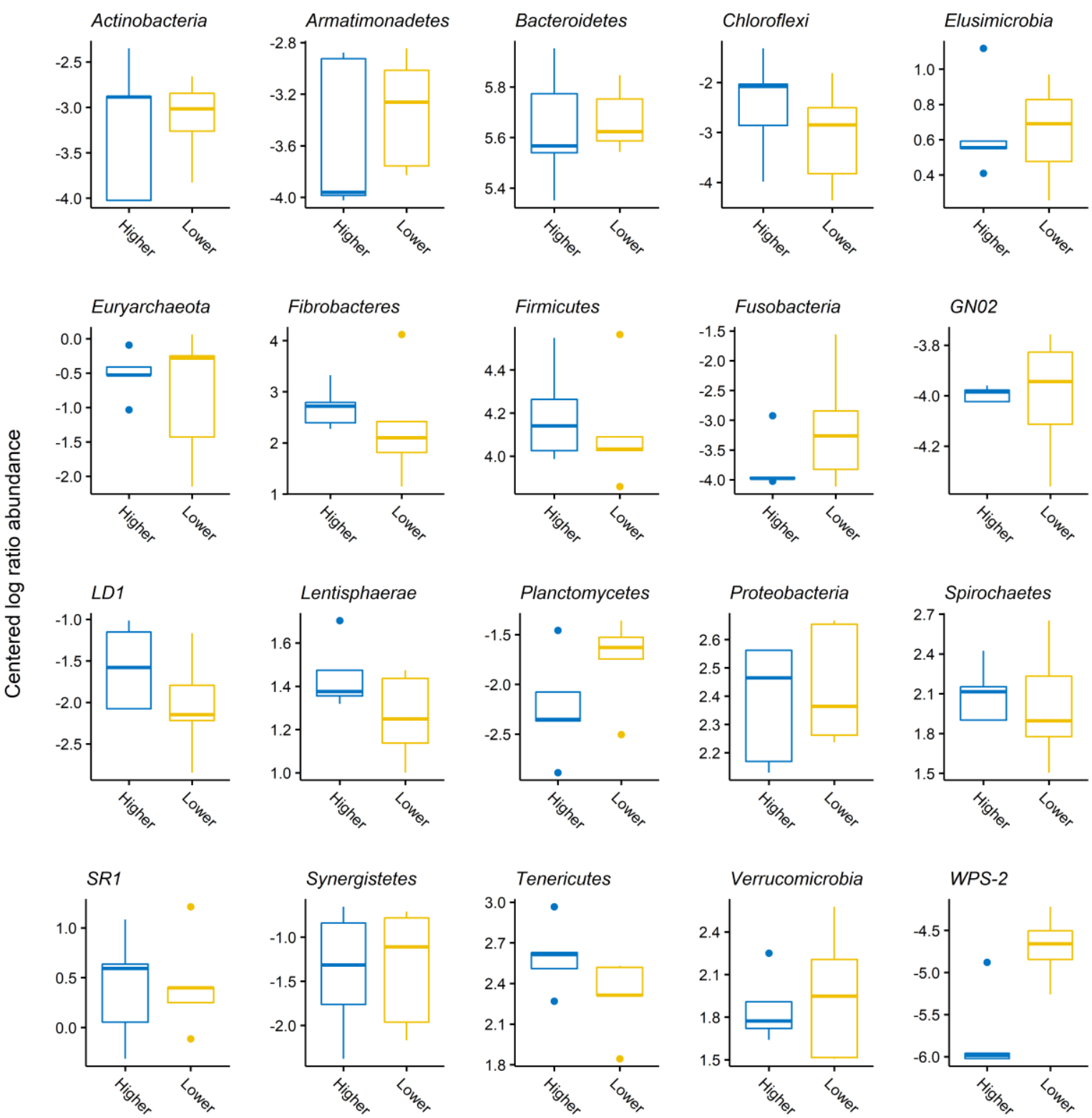

Classes of Average Dairy Gain of Weight (ADG)

Figure 2. Centered log ratio transformed abundance of all bacterial phyla present in ruminal samples from bovines with Higher ADG (yellow) and Lower ADG (blue). Differences were tested with the ALDEx package. No significant difference between groups was found (effect size $<1$, p-value $>0.1$ ). Note that negative clr values indicate very low abundance. 
The bacterial and fungal richness, at the Operational Taxonomic Unity (OTU) level, is presented in Figure 4. Both communities presented similar number of observed OTUs when comparing ADG classes. Although no differences were detected within this alpha diversity measurement, PERMANOVA outputs (Table 3) showed significant differences in bacterial composition between the two ADG classes.

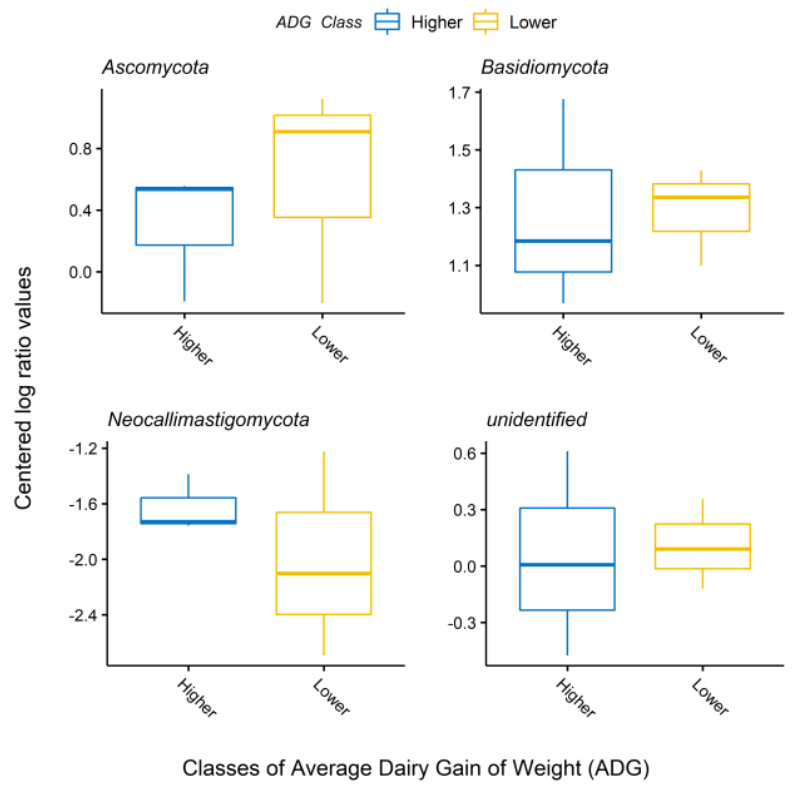

Figure 3. Centered log ratio transformed abundance of all fungal phyla present in ruminal samples from bovines with Higher ADG (yellow) and Lower ADG (blue). Differences were tested with the ALDEx package. No significant difference between groups was found (effect size $<1$, p-value $>0.1$ ). Note that negative clr values indicate very low abundance.
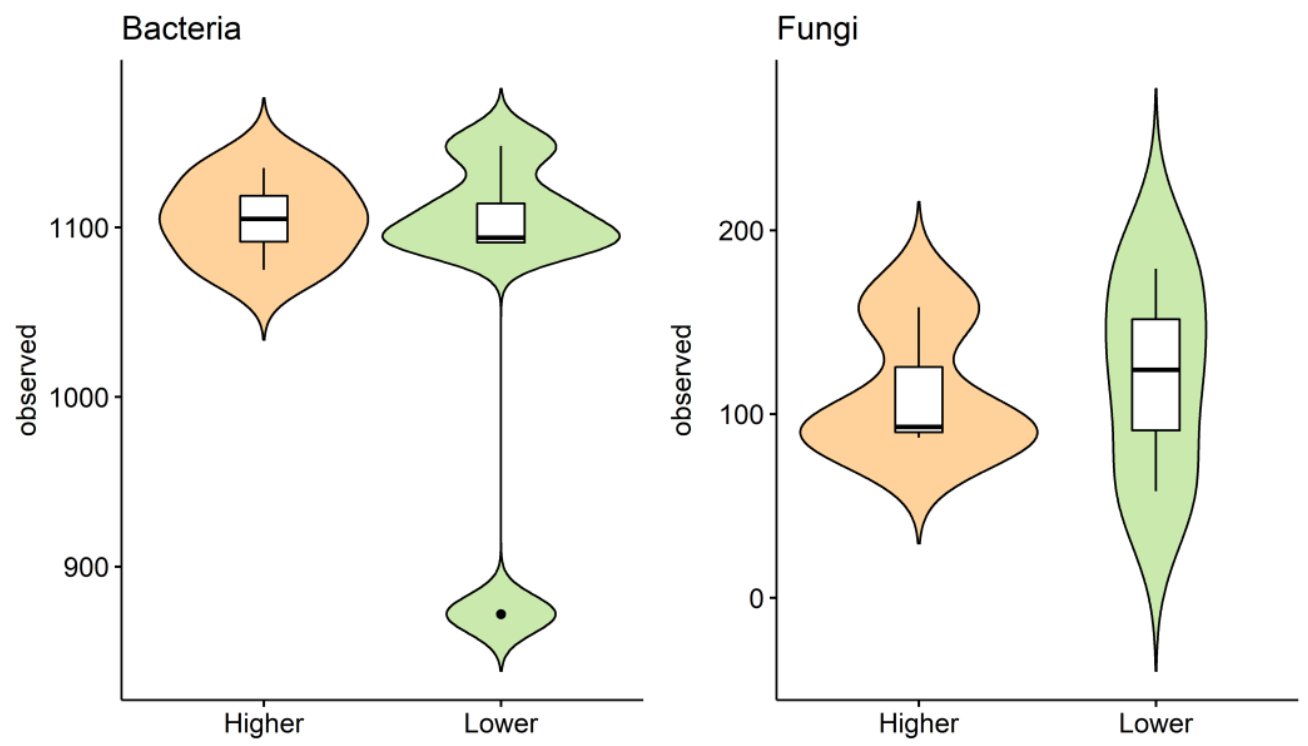

Classes of Average Dairy Gain of Weight (ADG)

Figure 4. Number of observed OTUs of bacterial (left panel) and fungal (right panel) communities in the Higher ADG and Lower ADG groups. Graphs represent the number of different operational taxonomic units (OTUs) found in each group. Boxes inside the violins span the first to third quartiles; the horizontal line inside the boxes represents the median. Whiskers extending vertically from the boxes indicate variability outside the upper and lower quartiles, and the single black circles indicate outliers. 


\subsection{Differential abundance analysis revealed microbial patterns associated with enhanced gain of weight}

Differential abundance analysis at the OTU level (using the highest possible taxonomy rank) reveled many microorganisms differentially abundant between ADG classes (Table 4). Three OTUs closest related to the genus RFN20 presented higher abundance in the Higher ADG group, as well as OTUs closest related to the genus Anaeroplasma and the family RF16. Two OTUs assigned as Prevotella genus presented higher abundance in higher ADG group, but another OTU also assigned as Prevotella presented opposite trend, with higher abundance in the lower ADG group. Among fungal OTUs, the most important taxa with differential abundance were the genera Aureobasidium, Cryptococcus and Sarocladium, all of them increased in the higher ADG group. In addition, OTUs closest related to the orders Tremellales and Pleosporales were also more abundant in the higher ADG group. The only OTU significantly more abundant in lower ADG group was assigned to the genus Ceratobasidium.

Table 4. List of microbial taxa with significant difference between higher and lower group of Average Daily Gain of Weight (ADG).

\begin{tabular}{|c|c|c|c|c|c|c|}
\hline & Taxon & rab.win.Lowera & rab.win.Higher ${ }^{b}$ & effect ${ }^{c}$ & overlap $^{d}$ & we.ep \\
\hline \multirow[t]{8}{*}{ Bacteria } & RFN20 (1) & 2.47 & 4.21 & 1.54 & 0.04 & 0.01 \\
\hline & RFN20 (2) & 2.68 & 4.18 & 1.28 & 0.10 & 0.03 \\
\hline & RFN20 (3) & 4.62 & 5.88 & 1.20 & 0.12 & 0.04 \\
\hline & Anaeroplasma & -2.74 & 1.82 & 1.54 & 0.04 & 0.03 \\
\hline & Prevotella (1) & -1.35 & 1.99 & 1.25 & 0.07 & 0.04 \\
\hline & Prevotella (2) & 1.61 & 3.01 & 1.10 & 0.09 & 0.05 \\
\hline & Prevotella (3) & 2.46 & 0.76 & -1.08 & 0.10 & 0.06 \\
\hline & $R F 16$ & -0.05 & 1.88 & 1.17 & 0.10 & 0.07 \\
\hline \multirow[t]{6}{*}{ Fungi } & Aureobasidium & -1.52 & 3.59 & 1.90 & 0.02 & 0.05 \\
\hline & $\begin{array}{l}\text { Tremellales fam } \\
\text { Incertae sedis }\end{array}$ & -1.14 & 6.41 & 1.70 & 0.03 & 0.06 \\
\hline & Ceratobasidium & 5.04 & -3.09 & -1.62 & 0.04 & 0.07 \\
\hline & Pleosporales & -1.35 & 4.65 & 1.71 & 0.02 & 0.07 \\
\hline & Cryptococcus & 0.31 & 6.07 & 1.60 & 0.00 & 0.09 \\
\hline & Sarocladium & -1.91 & 4.99 & 1.38 & 0.05 & 0.09 \\
\hline
\end{tabular}

${ }^{a}$ median centered log-ratio value for the Lower ADG group of samples; ${ }^{b}$ median centered log-ratio value for the Higher ADG group of samples; ${ }^{c}$ effect size of the difference, median of difference between groups on a $\log$ base 2 scale/largest median variation within groups, positive values indicate a higher abundance in the Higher ADG group whereas negative values indicate higher abundance in the lower ADG group; ${ }^{\mathrm{d}}$ confusion in assigning an observation to Higher or Lower group; e the expected value of the Welch Test $\mathrm{P}$ value. Table includes all OTUs with effect $>1$ and $p$-value $<0.1$.

\subsection{Testing the biomarkers with the entire dataset}

Our approach defined a group of microbial taxa differentially abundant in the Higher and Lower ADG groups. To confirm these findings, we correlated the average daily gain of weight with the relative abundance (normalized by centered log ratios) of each taxa from the Table 4 using all samples. The results indicate that, bacterial OTUs found here were associated with gain of weight in beef cattle (Figures 5 ).

OTUs closest related to RFN20 (1), Prevotella (1) and Anaeroplasma presented the highest and most confident positive correlations $(R>0.6$ and $p<0.01)$ with average daily gain of weight. 
These results indicate the abundance of each one of these OTUs can individually explain more than $60 \%$ of the ADG from tested animals with a confidence higher than $99 \%$.

Correlations between differential abundant fungal taxa and average dairy gain of weight were also confirmed using the entire dataset (Figure 6). Ceratobasidium provided the main (and only) negative correlation between fungal taxa and ADG $(R=-0.65)$, with high confidence $(p=0.023)$. On the other hand, the non-identified OTU closest related to the order Tremellales, provided the highest positive correlation with ADG $(R=0.67$ and $p=0.018)$. The genus Cryptococcus was also positively correlated with ADG $(\mathrm{R}=0.58$ and $\mathrm{p}=0.049)$. All other fungi previously identified as increased by the ALDEx analysis presented weaker correlations.

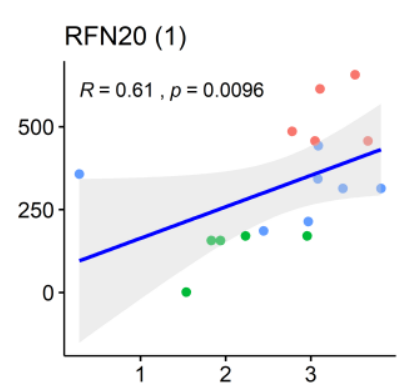

ADG Class - Higher - Lower - Middle
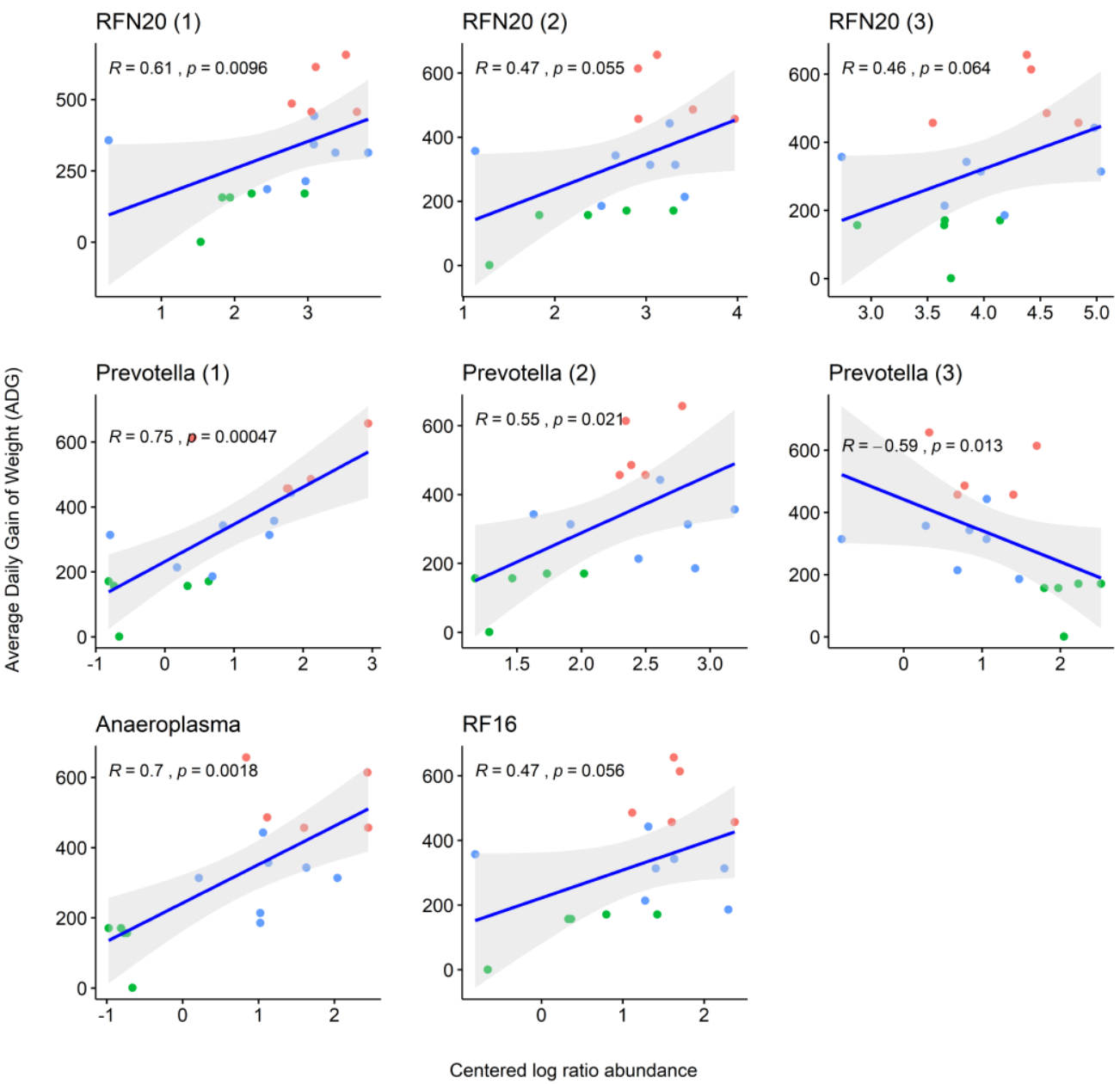

Figure 5. Ratios of OTUs closest related to the bacterial taxa and their correlation with daily gain of weight in beef cattle's rumen. 


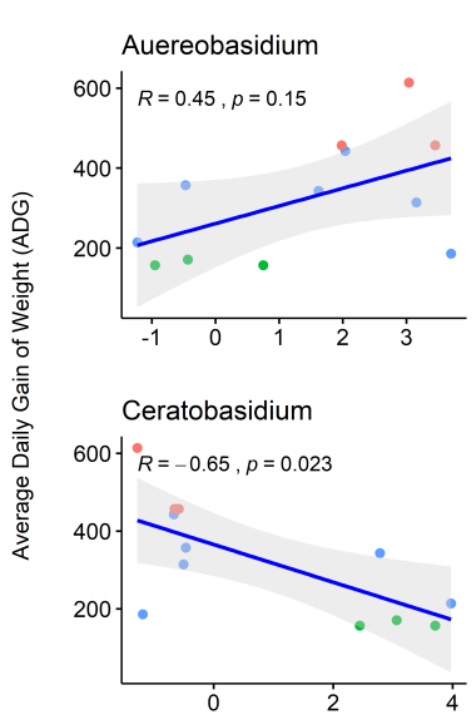

ADG Class - Higher - Lower - Middle
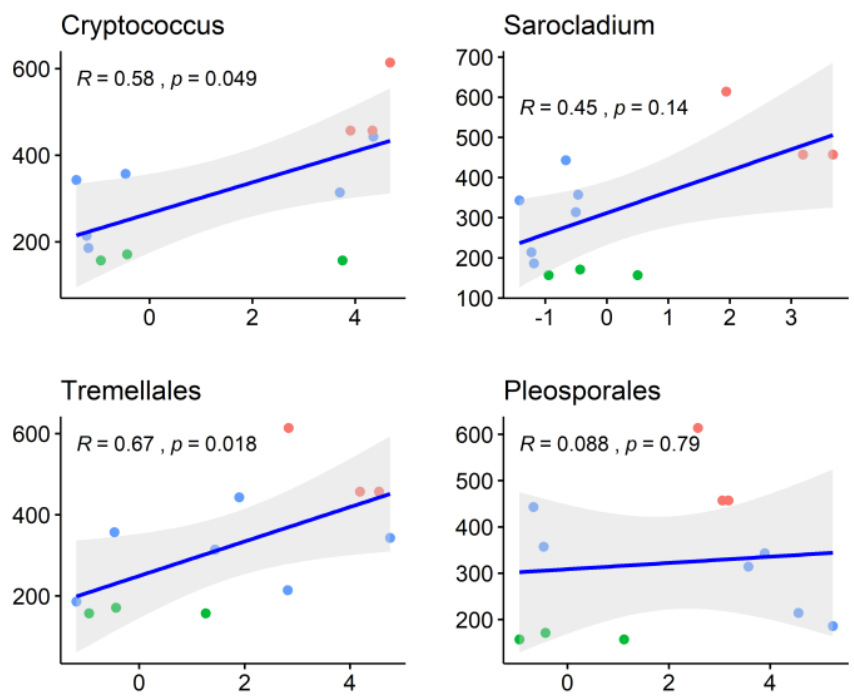

Centered log ratio abundance

Figure 6. Ratios of OTUs closest related to fungal taxa and their correlation with daily gain of weight in beef cattle's rumen.

\section{Discussion}

This study identified differences in microbial communities from rumen of heifers with increased weight gain. To our knowledge, it's the first work that attempted to measure these differences in heifers in natural grasslands from the Pampa Biome, a biome historically linked to cattle breeding $[32,33]$. Here, we presented multiple evidences supporting previous works about general structure of rumen microbiota and added knowledge about the association of microbes with weight gain, a key factor for beef production.

Once we established groups of animals with highly contrasting weight gain (Figure 1), kept for more than two years in the same conditions (Tables 1 and 2), we showed the overall community of heifers was similar in diversity of phyla (Figure 2 and 3) and in richness of microbial taxa (Figure 4), irrespective of the weigh gain. Several studies postulated the rumen microbiome presents a stable community $[2,15,17,34-36]$. These reports indicated a high dominance of Bacteroidetes species inhabiting the ruminal fluid within various environments and conditions, followed by a smaller, but consistent, presence of Firmicutes. Our results are aligned with those findings (Figure 2), as well as with findings about fungal samples which were not correlated to any dominant phyla in rumen neither in the literature nor in our work (Figure 3) [2,17,34,37].

Differences between ADG classes were tested at the OTU level. Results from PERMANOVA analyses (Table 3), indicated reliable differences between higher and lower ADG groups. In fact, analyzing bacterial samples at the OTU level, we showed a strong increment of various taxa associated to digestion processes in animals with higher ADG (Table 4). The unclassified RFN20, for example is a rumen-specific bacterium member of the subclass Erysipelotrichia, which was previously correlated to mice under high fat diets [38,39]. Additionally, Anaeroplasma is a genus characterized by its anaerobic fermentation which products fatty acids as propionate [40] whereas Prevotella produces majorly succinic and acetic acids [41]. All of them are short chain fatty acids important to the glucose biosynthesis in cattle [2]. Lastly, the family BF16 is also a rumen-native taxon with higher abundance in mature calves and also correlated to methane production [42,43]. All of these taxa were previously reported as members of the regular and efficient microbiota from rumen and their increased abundance may indicate an improved ability to digestion or, at least, a need for more specialized fermentation in rumen due to, for example, more food intake.

Nonetheless, the case of Prevotella OTUs deserves additional attention. Despite two OTUs assigned as Prevotella presented higher abundance in higher ADG samples, another OTU also related to the genus Prevotella was increased in the lower ADG group (Table 4, Figure 6). The Prevotella is a 
very complex genus and its species are can adapt to the most different environments [44]. In addition, this dubious role of ruminal Prevotella species in states of good or bad production were previously reported in milk production. Chiquette and colleagues, for example, showed that a strain of Prevotella bryantii acts as probiotic increasing the fat content in milk [45] whereas Jami and collaborators found an OTUs assigned as Prevotella negatively correlated with milk-fat yield [16]. For this reason, we believe that we found different Prevotella species that may be related to higher and lower weight gain.

Within the fungal communities, the three genera assigned as increased in higher AGD heifers present characteristics that may increase the animal's digestibility (Table 4). Aureobasidium is a genus of yeast-like fungi with a great genomic plasticity that allows it to adapt to the most different environments [46,47]. This genus produces a high number of Carbohydrate Active Enzymes (CAZy), which synthesize or breakdown saccharides and are very important for the ruminal digestion of organic matter [48]. Cryptococcus is a genus of soil-resident fungi correlated to degradation of wood and associated with bird excreta [49,50]. This curious association may be explained by the production of phenol oxidases, enzymes that increase the absorption of carbon and nitrogen besides acting in lignin degradation, carbon mineralization and dissolved organic carbon exportation [51]. Sarocladium, is a known phytopathogen specialized in secretion of enzymes that degrade the cell wall and other hard plant cellular components [52-54]. Additionally, Sarocladium is an endophytic of Poaceae species, the main family in the native Pampa grasslands [55].

Alongside the identified genera, we identified two OTUs also correlated to the higher weight gain only classified at the order level (Table 4). Tremelalles and Pleosporales represents a wide range of fungi from yeasts to filamentous fungi. Most of them are cosmopolitan saprophytes, but others are described living in vascular plants [56-59]. Because of this, we are not able to suggest biochemical mechanisms that explain the relationship among these families and a higher weight gain. Nonetheless, the aforementioned genus Cryptococcus is included in the order Tremellales [56], which may support the presence of it and/or other close related taxa to an increased weight gain.

On the other hand, we found an OTU closest related to the genus Ceratobasidium as increased in lower ADG group. This genus comprises a set of cosmopolitan fungi, most of them saprophytes in the soil [60]. Some strains of this genus, however, have been successfully tested as a tool for biocontrol of the plant pathogen Rhizoctonia solani [61]. These findings may suggest an action of Creatobasidium as a strong competitive microbe in the rumen environment of these animals and may lead to a low abundance of other microorganisms with phytopathogenic activities that may improve the digestion process, what is supported by its direct correlation with lower gain of weight (Figure 6).

In general, these findings suggested a core of microbes correlated to an increased weight gain in cattle. Either in bacteria or in fungi we found taxa associated with higher fermentation and digestion rates increased in higher ADG group. Most of them are known to be specialists in producing substances important for plant organic matter degradation, what increases the digestion and consequently, the absorption of nutrients and gain of weight. Alongside, the presence of these microorganisms may increase the food intake by cattle, once a faster digestion may lead to more opportunity for more food intake.

The use of microorganisms in cattle nutrition is not novel. However, the currently available products are focused in a narrow range of species, mainly yeasts from the genera Saccaromyces and Kluyveromyces [62]. Our results have found no significant correlation of these genera with weight gain. On the other hand, we found a yeast that is naturally present in rumen environment correlated with a higher weight gain: Aureobasidium. Its aforementioned production of CAZymes may be linked to a higher efficiency in degradation of plant carbohydrates and supports future studies to evaluate possible benefits that this genera can induce in beef cattle breeding. The same perspective is expected for the bacterial genus Prevotella, which have shown good results in production chain with some lineages [45] and for the genera Anaeroplasma and RFN20, both of them highly correlated with high weight gain (Figures 5 and 6 ) and may be important for cattle nutrition either individually or in a consortium. The study of Ceratobasidium, on the other hand, as a suppressor of weight gain may 
also be necessary, once this genus was pointed as strongly correlated with lower weight gain (Figure $6)$.

\section{Conclusions}

Here, we showed microbial patterns associated with increased weight gain in beef cattle from natural grasslands of the Brazilian Pampa. The results point to microorganisms with high ability to produce specific compounds related to a better digestion process. Those microorganism are not currently available in commercial products focused in improving cattle nutrition and might be focus of studies for the development of new and more efficient probiotic products. This report may also be important for future research for biomarkers of productivity and for microbial therapies to improve productivity in beef cattle causing less environmental damages.

Author Contributions: conceptualization, A.S.F, D.B.D, and L.F.W.R; methodology, A.S.F., D.B.D. and B.M.T.; validation, D.B.D; formal analysis, A.S.F and L.F.W.R; investigation, A.S.F., D.B.D. and B.M.T.; resources, D.B.D. and L.F.W.R; data curation, A.S.F and L.F.W.R.; writing - original draft preparation, A.S.F and D.B.D.; writing - review and editing, A.S.F, D.B.D and L.F.W.R; visualization, B.M.T; supervision, L.F.W.R; project administration, D.B.D and L.F.W.R.; funding acquisition, D.B.D and L.F.W.R".

Funding: This research was funded by the Coordenação de Aperfeiçoamento de Pessoal de Nível Superior-Brasil (CAPES) (Finance Code 001). L.F.W. Roesch, A.S. de Freitas and D.B. de David received research fellowships from the CNPq.

Conflicts of Interest: The authors declare no conflict of interest. The funders had no role in the design of the study; in the collection, analyses, or interpretation of data; in the writing of the manuscript, or in the decision to publish the results.

\section{References}

1. Malmuthuge, N.; Griebel, P.J.; Guan, L.L. Taxonomic Identification of Commensal Bacteria Associated with the Mucosa and Digesta throughout the Gastrointestinal Tracts of Preweaned Calves. Applied and Environmental Microbiology 2014, 80, 2021-2028.

2. Wirth, R.; Kádár, G.; Kakuk, B.; Maróti, G.; Bagi, Z.; Szilágyi, Á.; Rákhely, G.; Horváth, J.; Kovács, K.L. The Planktonic Core Microbiome and Core Functions in the Cattle Rumen by Next Generation Sequencing. Frontiers in Microbiology 2018, 9 .

3. Linn, J.; Hutjens, M.; Shaver, R.; Otterby, D.; Howard, W.T.; Kilmer, L. The ruminant digestive system. University of Minnesota Extension 2018.

4. Parish, J.; Rivera, D. Understanding the Ruminant Animal Digestive System. Mississippi State University 2017, 8 .

5. Mccann, J.C.; Wickersham, T.A.; Loor, J.J. High-throughput Methods Redefine the Rumen Microbiome and Its Relationship with Nutrition and Metabolism. Bioinformatics and Biology Insights 2014, 8, BBI.S15389.

6. Myer, P.R.; Kim, M.; Freetly, H.C.; Smith, T.P.L. Evaluation of $16 \mathrm{~S}$ rRNA amplicon sequencing using two next-generation sequencing technologies for phylogenetic analysis of the rumen bacterial community in steers. Journal of Microbiological Methods 2016, 127, 132-140.

7. Henderson, G.; Ganesh, S.; Jonker, A.; Young, W.; Global Rumen Census Collaborators; Janssen, P.H. Rumen microbial community composition varies with diet and host, but a core microbiome is found across a wide geographical range. Scientific Reports 2015, 5.

8. Jami, E.; Mizrahi, I. Composition and Similarity of Bovine Rumen Microbiota across Individual Animals. PLOS ONE 2012, 7, e33306. 
9. Jesus, R.B. de; Omori, W.P.; Lemos, E.G.D.M.; Souza, J.A.M. de Bacterial diversity in bovine rumen by metagenomic 16S rDNA sequencing and scanning electron microscopy. Acta Scientiarum. Animal Sciences 2015, $37,251$.

10. Nathani, N.M.; Kothari, R.K.; Patel, A.K.; Joshi, C.G. Functional Characterization Reveals Novel Putative Coding Sequences in Prevotella ruminicola Genome Extracted from Rumen Metagenomic Studies. Journal of Molecular Microbiology and Biotechnology 2015, 25, 292-299.

11. Fliegerova, K.; Kaerger, K.; Kirk, P.; Voigt, K. Rumen Fungi. In Rumen Microbiology: From Evolution to Revolution; Puniya, A.K., Singh, R., Kamra, D.N., Eds.; Springer India: New Delhi, 2015; pp. 97-112 ISBN 978-81-322-2400-6.

12. Song, J.; Jeong, J.Y.; Kim, M. Diversity Census of Fungi in the Ruminal Microbiome: A meta-analysis. Journal of the Korea Academia-Industrial Cooperation Society 2017, 18, 466-472.

13. Carberry, C.A.; Kenny, D.A.; Han, S.; McCabe, M.S.; Waters, S.M. Effect of Phenotypic Residual Feed Intake and Dietary Forage Content on the Rumen Microbial Community of Beef Cattle. Applied and Environmental Microbiology 2012, 78, 4949-4958.

14. Ishaq, S.L.; AlZahal, O.; Walker, N.; McBride, B. An Investigation into Rumen Fungal and Protozoal Diversity in Three Rumen Fractions, during High-Fiber or Grain-Induced Sub-Acute Ruminal Acidosis Conditions, with or without Active Dry Yeast Supplementation. Frontiers in Microbiology 2017, 8.

15. Indugu, N.; Vecchiarelli, B.; Baker, L.D.; Ferguson, J.D.; Vanamala, J.K.P.; Pitta, D.W. Comparison of rumen bacterial communities in dairy herds of different production. BMC Microbiology 2017, 17.

16. Jami, E.; White, B.A.; Mizrahi, I. Potential Role of the Bovine Rumen Microbiome in Modulating Milk Composition and Feed Efficiency. PLoS ONE 2014, 9, e85423.

17. Stewart, R.D.; Auffret, M.D.; Warr, A.; Walker, A.W.; Roehe, R.; Watson, M. Compendium of 4,941 rumen metagenome-assembled genomes for rumen microbiome biology and enzyme discovery. Nature Biotechnology 2019, 37, 953-961.

18. Lancaster, R.J. Estimation of Digestibility of Grazed Pasture from Fæces Nitrogen. Nature 1949, 163, 330-331.

19. Rosa, F.Q.; Pagel, R.; David, D.B.; da Trindade, J.K.; Castagnara, D.D.; Bremm, C.; Azevedo, E.B. Measurement of nutritional parameters by fecal markers in cattle fed heterogeneous natural grasslands. In Press.

20. Pylro, V.S.; Roesch, L.F.W.; Ortega, J.M.; do Amaral, A.M.; Tótola, M.R.; Hirsch, P.R.; Rosado, A.S.; Góes-Neto, A.; da Costa da Silva, A.L.; Rosa, C.A.; et al. Brazilian Microbiome Project: Revealing the Unexplored Microbial Diversity -Challenges and Prospects. Microbial Ecology 2014, 67, 237-241.

21. Caporaso, J.G.; Lauber, C.L.; Walters, W.A.; Berg-Lyons, D.; Lozupone, C.A.; Turnbaugh, P.J.; Fierer, N.; Knight, R. Global patterns of $16 \mathrm{~S}$ rRNA diversity at a depth of millions of sequences per sample. Proceedings of the National Academy of Sciences 2011, 108, 4516-4522.

22. GARDES, M.; BRUNS, T.D. ITS primers with enhanced specificity for basidiomycetes - application to the identification of mycorrhizae and rusts. Molecular Ecology 1993, 2, 113-118.

23. Walters, W.; Hyde, E.R.; Berg-Lyons, D.; Ackermann, G.; Humphrey, G.; Parada, A.; Gilbert, J.A.; Jansson, J.K.; Caporaso, J.G.; Fuhrman, J.A.; et al. Improved Bacterial 16S rRNA Gene (V4 and V4-5) and Fungal Internal Transcribed Spacer Marker Gene Primers for Microbial Community Surveys. mSystems 2016, 1. 
24. White, T.J.; Bruns, T.D.; Lee, S.B.; Taylor, J.W. Amplification and direct sequencing of fungal ribosomal RNA Genes for phylogenetics. In PCR - Protocols and Applications - A Laboratory Manual; Academic Press, 1990; pp. 315-322 ISBN 0-12-372180-6.

25. Hamady, M.; Walker, J.J.; Harris, J.K.; Gold, N.J.; Knight, R. Error-correcting barcoded primers for pyrosequencing hundreds of samples in multiplex. Nature Methods 2008, 5, 235-237.

26. Pylro, V.S.; Roesch, L.F.W.; Morais, D.K.; Clark, I.M.; Hirsch, P.R.; Tótola, M.R. Data analysis for 16S microbial profiling from different benchtop sequencing platforms. Journal of Microbiological Methods 2014, 107, 30-37.

27. Gloor, G.B.; Reid, G. Compositional analysis: a valid approach to analyze microbiome high-throughput sequencing data. Canadian Journal of Microbiology 2016, 62, 692-703.

28. Dixon, P. VEGAN, a package of R functions for community ecology. Journal of Vegetation Science 2003, 14, 927-930.

29. Gloor, G. ANOVA-Like Differential Expression tool for high throughput sequencing data. 19.

30. Gloor, G.B.; Macklaim, J.M.; Fernandes, A.D. Displaying Variation in Large Datasets: Plotting a Visual Summary of Effect Sizes. Journal of Computational and Graphical Statistics 2016, 25, 971-979.

31. Pourhoseingholi, M.A.; Baghestani, A.R.; Vahedi, M. How to control confounding effects by statistical analysis. Gastroenterology and Hepatology From Bed to Bench 2012, 5, 79-83.

32. Roesch, L.F.; Vieira, F.; Pereira, V.; Schünemann, A.L.; Teixeira, I.; Senna, A.J.; Stefenon, V.M. The Brazilian Pampa: A Fragile Biome. Diversity 2009, 1, 182-198.

33. Rouse, J.E. The criollo: Spanish cattle in the Americas.; University of Oklahoma Press., 1977; ISBN 0-8061-1404-5.

34. Malmuthuge, N.; Guan, L.L. Understanding host-microbial interactions in rumen: searching the best opportunity for microbiota manipulation. Journal of Animal Science and Biotechnology 2017, 8 .

35. Sun, H.-Z.; Xue, M.; Guan, L.L.; Liu, J. A collection of rumen bacteriome data from 334 mid-lactation dairy cows. Scientific Data 2019, 6.

36. Tapio, I.; Shingfield, K.J.; McKain, N.; Bonin, A.; Fischer, D.; Bayat, A.R.; Vilkki, J.; Taberlet, P.; Snelling, T.J.; Wallace, R.J. Oral Samples as Non-Invasive Proxies for Assessing the Composition of the Rumen Microbial Community. PLOS ONE 2016, 11, e0151220.

37. Akin, D.E.; Borneman, W.S. Role of Rumen Fungi in Fiber Degradation. Journal of Dairy Science 1990, 73, 3023-3032.

38. Greiner, T.; Bäckhed, F. Effects of the gut microbiota on obesity and glucose homeostasis. Trends in Endocrinology \& Metabolism 2011, 22, 117-123.

39. Turnbaugh, P.J.; Ridaura, V.K.; Faith, J.J.; Rey, F.E.; Knight, R.; Gordon, J.I. The Effect of Diet on the Human Gut Microbiome: A Metagenomic Analysis in Humanized Gnotobiotic Mice. Science Translational Medicine 2009, 1, 6ra14-6ra14.

40. Brown, D.R.; Bradbury, J.M.; Johansson, K.-E. Anaeroplasma. In Bergey's Manual of Systematics of Archaea and Bacteria; Whitman, W.B., Rainey, F., Kämpfer, P., Trujillo, M., Chun, J., DeVos, P., Hedlund, B., Dedysh, S., Eds.; John Wiley \& Sons, Ltd: Chichester, UK, 2015; pp. 1-5 ISBN 978-1-118-96060-8.

41. Shah, H.N.; Chattaway, M.A.; Rajakurana, L.; Gharbia, S.E. Prevotella. In Bergey's Manual of Systematics of Archaea and Bacteria; American Cancer Society, 2015; pp. 1-25 ISBN 978-1-118-96060-8. 
42. Cunha, C.S.; Marcondes, M.I.; Veloso, C.M.; Mantovani, H.C.; Pereira, L.G.R.; Tomich, T.R.; Dill-McFarland, K.A.; Suen, G. Compositional and structural dynamics of the ruminal microbiota in dairy heifers and its relationship to methane production. Journal of the Science of Food and Agriculture 2019, 99, $210-218$. 43. Smith, K.E.; Garza, A.L.; Butterfield, K.M.; Dickey, A.M.; Lindholm-Perry, A.K.; Wells, J.E.; Freetly, H.C.; Lodge-Ivey, S.L. Succession of ruminal bacterial species and fermentation characteristics in preweaned Brangus calves1. Translational Animal Science 2018, 2, S48-S52.

44. Gupta, V.K.; Chaudhari, N.M.; Iskepalli, S.; Dutta, C. Divergences in gene repertoire among the reference Prevotella genomes derived from distinct body sites of human. BMC Genomics 2015, 16, 153-153.

45. Chiquette, J.; Allison, M.J.; Rasmussen, M.A. Prevotella bryantii 25A Used as a Probiotic in Early-Lactation Dairy Cows: Effect on Ruminal Fermentation Characteristics, Milk Production, and Milk Composition. Journal of Dairy Science 2008, 91, 3536-3543.

46. Gostinčar, C.; Grube, M.; Gunde-Cimerman, N. Evolution of Fungal Pathogens in Domestic Environments? Fungal Biology 2011, 115, 1008-1018.

47. Slepecky, R.A.; Starmer, W.T. Phenotypic plasticity in fungi: a review with observations on Aureobasidium pullulans. Mycologia 2009, 101, 823-832.

48. Gostinčar, C.; Ohm, R.A.; Kogej, T.; Sonjak, S.; Turk, M.; Zajc, J.; Zalar, P.; Grube, M.; Sun, H.; Han, J.; et al. Genome sequencing of four Aureobasidium pullulans varieties: biotechnological potential, stress tolerance, and description of new species. BMC Genomics 2014, 15, 549.

49. Kobayashi, C.C.B.A.; Souza, L.K.H. e; Fernandes, O. de F.L.; Brito, S.C.A. de; Silva, A.C.; Sousa, E.D. de; Silva, M. do R.R. Characterization of Cryptococcus neoformans isolated from urban environmental sources in Goiânia, Goiás State, Brazil. Revista do Instituto de Medicina Tropical de São Paulo 2005, 47, 203-207.

50. Refai, M.K.; El-Hariri, M.; Alarousy, R. Cryptococcosis in Animals and Birds: A Review. 2017, 22.

51. Sinsabaugh, R.L. Phenol oxidase, peroxidase and organic matter dynamics of soil. Soil Biology and Biochemistry 2010, 42, 391-404.

52. Giraldo, A.; Gené, J.; Sutton, D.A.; Madrid, H.; de Hoog, G.S.; Cano, J.; Decock, C.; Crous, P.W.; Guarro, J. Phylogeny of $<\mathrm{I}>$ Sarocladium $</ \mathrm{I}>(<\mathrm{I}>$ Hypocreales $</ \mathrm{I}>)$. Persoonia - Molecular Phylogeny and Evolution of Fungi $2015,34,10-24$.

53. Guimarães, R.A.; Lobo, V.L. da S.; Côrtes, M.V.C.B.; Filippi, M.C.C. de; Prabhu, A.S. Characterization of Sarocladium oryzae and its reduction potential of rice leaf blast. Pesquisa Agropecuária Tropical 2017, 47, 41-52.

54. Hou, Y.M.; Zhang, X.; Zhang, N.N.; Naklumpa, W.; Zhao, W.Y.; Liang, X.F.; Zhang, R.; Sun, G.Y.; Gleason, M.L. Genera Acremonium and Sarocladium Cause Brown Spot on Bagged Apple Fruit in China. Plant Disease 2019, 103, 1889-1901.

55. Liu, X.B.; Guo, Z.K.; Huang, G.X. Sarocladium brachiariae sp. nov., an endophytic fungus isolated from Brachiaria brizantha. Mycosphere 2017, 8, 827-834.

56. Findley, K.; Rodriguez-Carres, M.; Metin, B.; Kroiss, J.; Fonseca, Á.; Vilgalys, R.; Heitman, J. Phylogeny and Phenotypic Characterization of Pathogenic Cryptococcus Species and Closely Related Saprobic Taxa in the Tremellales. Eukaryotic Cell 2009, 8, 353-361.

57. Shearer, C.A.; Raja, H.A.; Miller, A.N.; Nelson, P.; Tanaka, K.; Hirayama, K.; Marvanová, L.; Hyde, K.D.; Zhang, Y. The molecular phylogeny of freshwater Dothideomycetes. Studies in Mycology 2009, 64, $145-153$.

58. Suetrong, S.; Schoch, C.L.; Spatafora, J.W.; Kohlmeyer, J.; Volkmann-Kohlmeyer, B.; Sakayaroj, J.; Phongpaichit, S.; Tanaka, K.; Hirayama, K.; Jones, E.B.G. Molecular systematics of the marine Dothideomycetes. Studies in Mycology 2009, 64, 155-173. 
59. Zhang, Y.; Schoch, C.L.; Fournier, J.; Crous, P.W.; de Gruyter, J.; Woudenberg, J.H.C.; Hirayama, K.; Tanaka, K.; Pointing, S.B.; Spatafora, J.W.; et al. Multi-locus phylogeny of Pleosporales: a taxonomic, ecological and evolutionary re-evaluation. Studies in Mycology 2009, 64, 85-102.

60. McMahon, P.; Purwantara, A. Vascular Streak Dieback (Ceratobasidium theobromae): History and Biology. In Cacao Diseases: A History of Old Enemies and New Encounters; Bailey, B.A., Meinhardt, L.W., Eds.; Springer International Publishing: Cham, 2016; pp. 307-335 ISBN 978-3-319-24789-2.

61. Mosquera-Espinosa, A.T.; Bayman, P.; Prado, G.A.; Gómez-Carabalí, A.; Otero, J.T. The double life of Ceratobasidium : orchid mycorrhizal fungi and their potential for biocontrol of Rhizoctonia solani sheath blight of rice. Mycologia 2013, 105, 141-150.

62. Shurson, G.C. Yeast and yeast derivatives in feed additives and ingredients: Sources, characteristics, animal responses, and quantification methods. Animal Feed Science and Technology 2018, 235, 60-76. 